VOL. $6(1972), 11-18$.

\title{
Error bounds in the approximation of functions
}

\section{Badri N. Sahney and V. Venu Gopal Rao}

Let $f(x) \in \operatorname{Lip} \alpha, 0<\alpha<1$, in the range $(-\pi, \pi)$, and periodic with period $2 \pi$, outside this range. Also let

$$
f(x) \sim \frac{1}{2} a_{0}+\sum_{\nu=1}^{\infty}\left(a_{\nu} \cos v x+b_{\nu} \sin v x\right) \equiv \sum_{\nu=0}^{\infty} A_{\nu}(x) .
$$

We define the norm as

$$
\|f\|_{p}=\left\{\int_{0}^{2 \pi}|f(x)|^{p} d x\right\}^{1 / p}, p \geq 1
$$

and let the degree of approximation be given by

$$
E_{n}^{*}(f)=\min _{T_{n}}\left\|f-T_{n}\right\|_{p}
$$

where $T_{n}(x)$ is some $n$-th trigonometric polynomial.

We define a generating sequence $\left\{p_{n}\right\}$ such that it is non-negative, non-increasing and

$$
P(n)=p_{0}+p_{1}+\ldots+p_{n} \rightarrow \infty \text { as } n \rightarrow \infty .
$$

Approximation of functions belonging to the class Lip $\alpha$ by the $(c, \delta), 0<\delta \leq 1$, mean of its Fourier series is due to Chapman and Riesz. The following is the main result of our paper:

THEOREM. If $f(x)$ is periodic and belongs to the class $\operatorname{Lip}(\alpha, p), 0<\alpha \leq 1$, and if the sequence $\left\{p_{n}\right\}$ is defined 
as in (**), and if

$$
\left(\int_{1}^{n} \frac{(P(y))^{q}}{y^{q \alpha+2-q}} d y\right)^{1 / q}=O\left(P(n) / n^{\alpha+1 / q-1}\right),
$$

then

$$
E_{n}^{*}(f)=\min _{N_{n}}\left\|f-N_{n}\right\|_{p}=O\left(1 / n^{\alpha}\right)
$$

wnere $N_{n}(x)$ is the $\left(N, P_{n}\right)$ mean of the Fourier series (*) and, in particuzar, $T_{n}(x)=N_{n}(x)$.

We define the norm as

$$
\|f\|_{p}=\left\{\int_{0}^{2 \pi}|f(x)|^{p} d x\right\}^{1 / p}, \quad p \geq 1,
$$

and let the degree of approximation be given by (see [7])

$$
E_{n}^{*}(f)=\min _{T_{n}}\left\|f-T_{n}\right\|_{p} .
$$

Here $T_{n}(x)$ is some $n$-th trigonometric polynomial.

Let $f(x) \in$ Lip $\alpha, 0<\alpha<1$, in the interval $(-\pi, \pi)$, and periodic with period $2 \pi$ outside this range. Also let

$$
\begin{aligned}
f(x) & \sim \frac{1}{2} a_{0}+\sum_{v=1}^{\infty}\left(a_{v} \cos v_{x}+b_{\nu} \sin v x\right) \\
& =\sum_{\nu=0}^{\infty} A_{\nu}(x) .
\end{aligned}
$$

We write

$$
\phi(x, t)=\frac{f(x+t)+f(x-t)}{2}-f(x) .
$$


The following theorems are known:

THEOREM A. If the periodic function $f(x)$ belongs to the class Lipa, for $0<\alpha<1$, then the $(c, \delta)$ mean of its Fourier series for $0<\alpha<\delta \leq 1$, gives

$$
\max _{0 \leq x \leq 2 \pi}\left|f(x)-\sigma_{n}^{\delta}(x)\right|=O\left(1 / n^{\alpha}\right),
$$

and for $0<\alpha \leq \delta \leq 1$ satisfies

$$
\max _{0 \leq x \leq 2 \pi}\left|f(x)-\sigma_{n}^{\delta}(x)\right|=o\left(\log n / n^{\alpha}\right),
$$

where $\sigma_{n}^{\delta}(x)$ is the $(c, \delta)$ mean of the partial sum of (1.3).

THEOREM B. If the periodic function $f(x)$ belongs to the class Lip $\alpha$, for $0<\alpha \leq 1$, then the $(c, 1)$ mean of its Fourier series is given by

$$
\frac{1}{n} \sum_{k=1}^{n}\left|f(x)-S_{k}(x)\right|=O\left(\log n / n^{\alpha}\right),
$$

where $s_{k}$ is the partial sum of (1.3).

It is known [3] that for $\alpha=1$ the order of (2.3) is not $O(1 / n)$.

Theorem A was proved by Chapman and Riesz (see [1]) independently. Theorem $B$ is a simplified form of the result due to Alexits and Leindler [3]. Later Alexits and Králik [2] changed the summation in (2.3) from $k=n$ to $k=2 n-1$, along with some other improvements.

Let $\left\{p_{n}\right\}$ be a non-negative, non-increasing generating sequence for the $\left(N, p_{n}\right)$ method such that

$$
P_{n} \equiv P(n)=p_{0}+p_{1}+\ldots+p_{n} \rightarrow \infty \text { as } n \rightarrow \infty .
$$

Some of the related recent work on the Ijörlund method $\left(N, p_{n}\right)$ is due to Izumi and Izumi [4] and Sahney [6].

The object of this paper is to prove the following theorems:

THEOREM 1. If $f(x)$ is periodic and belongs to the class $\operatorname{Lip}(\alpha, p)$ 
for $0<\alpha<1$, such that $0<\alpha<\delta \leq 1$, then

$$
\begin{aligned}
E_{n}^{*}(f) & =\min _{T_{n}}\left\|f-\sigma_{n}^{(\delta)}\right\|_{p} \\
& =o\left(1 / n^{\alpha-1 / p}\right)
\end{aligned}
$$

where $\sigma_{n}^{(\delta)}(x)$ is the $(c, \delta)$ mean of (1.3). Here, in particular, $T_{n}(x)=\sigma_{n}^{\delta}(x)$

THEOREM 2. If $f(x)$ is periodic and belongs to the class Lip $(\alpha, p)$ for $0<\alpha \leq 1$, and if the sequence $\left\{p_{n}\right\}$ is as defined in (2.4) with the other requirements therein and if

$$
\left(\int_{1}^{n} \frac{(P(y))^{q}}{y^{\alpha+2-q}} d y\right)^{1 / q}=o\left(\frac{P(n)}{n^{\alpha+1 / q-1}}\right) \text {, }
$$

then

$$
\begin{aligned}
E_{n}^{*}(f) & =\min _{T_{n}}\left\|f-N_{n}\right\|_{p} \\
& =o\left(\frac{1}{n}\right),
\end{aligned}
$$

where $N_{n}(x)$ is the $\left(N, p_{n}\right)$ mean of (1.3), and, in particular, $T_{n}(x)=N_{n}(x)$.

3.

Proof of Theorem 1. Following Zygmund [7] we can write

$$
\text { (3.1) } \begin{aligned}
f(x)-\sigma_{n}^{(\delta)}(x) & =\frac{1}{\pi A} \int_{n}^{\pi} \phi(t) \sum_{k=0}^{n} \frac{A_{k}^{\delta-1} \sin k t}{t} d t+o(1) \\
& =\frac{1}{\pi A_{n}^{\delta}}\left[\int_{0}^{\pi / n}+\int_{\pi / n}^{\pi}\right] \phi(t) \sum_{k=0}^{n} \frac{A_{k}^{\delta-1} \sin k t}{t} d t+o(1) \\
& =I_{1}+I_{2}+o(1), \text { say. }
\end{aligned}
$$

By Hölder's inequality and the fact that $\phi(x) \in \operatorname{Lip}(\alpha, p)$, we get 
(3.2) $\quad I_{1}=\frac{1}{\pi A_{n}^{\delta}} \int_{0}^{\pi / n} \frac{\phi(t)}{t^{\alpha}} \sum_{k=0}^{n} \frac{A_{k}^{\delta-1} \sin k t}{t^{1-\alpha}} d t$

$$
\begin{aligned}
& \left.=\left.O\left(\frac{1}{\delta}\right)\left(\int_{0}^{\pi / n}\left|\frac{\phi(t)}{t^{\alpha}}\right|^{p} d t\right)^{1 / p}\left|\int_{0}^{\pi / n}\right| \sum_{k=0}^{n} \frac{A_{k}^{\delta-1} \sin k t}{t^{1-\alpha}}\right|^{q} d t\right)^{1 / q} \\
& =O\left(\frac{1}{\delta}\right) \cdot O(1)\left\{\int_{0}^{\pi / n}\left(a\left(\frac{n^{\delta} \cdot n t}{1-\alpha}\right)\right)^{q} d t\right\}^{1 / q} \\
& =O(n)\left(\int_{0}^{\pi / n} t^{\alpha q} d t\right)^{1 / q} \\
& =O(n)\left(\left[t^{1+\alpha q}\right]_{0}^{\pi / n}\right)^{1 / q} \\
& =O\left(\frac{1}{n^{\alpha-(1-1 / q)}}\right) \\
& =O\left(\frac{1}{n^{\alpha-1 / p}}\right)
\end{aligned}
$$

where $1 / p+1 / q=1$ such that $1 \leq p \leq \infty$.

Also, similarly, as above

$$
\begin{aligned}
& I_{2}=o\left(\frac{1}{n} \delta\right)\left(\int_{\pi / n}^{\pi}\left|\frac{\phi(t)}{t^{\alpha}}\right|^{p} d t\right)^{1 / p}\left(\int_{\pi / n}^{\pi}\left|\sum_{k=0}^{n} \frac{A_{k}^{\delta-1} \sin k t}{t^{1-\alpha}}\right|^{q} d t\right)^{1 / q} \\
& =O\left(\frac{1}{n}\right) O(1)\left(\int_{\pi / n}^{\pi}\left(\frac{1}{t}\right)^{\delta q} \frac{d t}{t^{(1-\alpha) q}}\right)^{1 / q} \\
& =o\left(\frac{1}{n} \delta\right)\left(\int_{\pi / n}^{\pi} \frac{d t}{t^{q+\delta q-\alpha q}}\right)^{1 / q} \\
& =o\left(\frac{1}{n} \delta\right)\left(\left[t^{1-q+\alpha q-\delta q}\right]_{\pi / n}^{\pi}\right)^{1 / q} \\
& =o\left(\frac{1}{n^{\delta}}\right)+o\left(\frac{1}{n}\right)\left(\frac{1}{n^{1 / q-1+\alpha-\delta}}\right) \\
& =o\left(\frac{1}{n^{\delta}}\right)+o\left(\frac{1}{n^{\alpha-1 / \tilde{E}}}\right) \text {. }
\end{aligned}
$$

Hence 


$$
I=o\left(\frac{1}{n^{\alpha-1 / p}}\right)+o\left(\frac{1}{\delta}\right)+o\left(\frac{1}{n^{\alpha-1 / p}}\right) .
$$

Therefore

$$
\begin{aligned}
E_{n}^{*}(f) & =\min _{\sigma_{n}^{(\delta)}}\left\|f-\sigma_{n}^{\delta}\right\|_{p} \\
& =o\left(1 / n^{\alpha-1 / p}\right) .
\end{aligned}
$$

4.

Proof of Theorem 2. Following Zygmund [7] we write

$$
\text { (4.1) } \begin{aligned}
f(x)-N_{n}(x) & =\frac{2}{\pi P(n)} \int_{0}^{\pi} \frac{\phi(t)}{t} \sum_{k=0}^{n} p_{k} \sin (n-k) t d t+o(1) \\
& =\frac{2}{\pi P(n)}\left(\int_{0}^{\pi / n}+\int_{\pi / n}^{\pi}\right) \frac{\phi(t)}{t} \sum_{k=0}^{n} p_{k} \sin (n-k) t d t+o(1) \\
& =J_{1}+J_{2}+o(1) \text {, say. }
\end{aligned}
$$

Applying Hölder's inequality and then by the fact that $\phi(x) \in \operatorname{Lip}(\alpha, p)$, we have

$$
\text { (4.2) } \begin{aligned}
J_{1} & =O\left(\frac{1}{P(n)}\right)\left(\int_{0}^{\pi / n}\left|\frac{\phi(t)}{t^{\alpha}}\right|^{p} d t\right)^{1 / p}\left(\int_{0}^{\pi / n}\left|\frac{\sum_{k=0}^{n} p_{k} \sin (n-k) t}{t^{1-\alpha}}\right| d t\right)^{1 / q} \\
& =O\left(\frac{1}{P(n)}\right) \cdot O(1)\left\{\int_{0}^{\pi / n}\left(\frac{O(P(n) \cdot n t)}{t^{1-\alpha}}\right)^{q} d t\right\}^{1 / q} \\
& =O(n)\left(\left[t^{\alpha q+1}\right]_{0}^{\pi / n}\right)^{1 / q} \\
& =O(n) \cdot O\left(1 / n^{\alpha+1 / q}\right) \\
& =O\left(1 / n^{\alpha+(1 / q-1)}\right) \\
& =O\left(1 / n^{\alpha-1 / p}\right) .
\end{aligned}
$$

Similarly 


$$
\text { (4.3) } \begin{aligned}
J_{2} & =O\left(\frac{1}{P(n)}\right)\left(\int_{\pi / n}^{\pi}\left|\frac{\phi(t)}{t^{\alpha}}\right|^{p} d t\right)^{1 / p}\left(\int_{\pi / n}^{\pi}\left|\frac{\sum_{k=0}^{n} p_{k} \sin (n-k) t}{t^{1-\alpha}}\right|^{q} d t\right)^{1 / q} \\
& =O\left(\frac{1}{P(n)}\right) \cdot O(1)\left(\int_{\pi / n}^{\pi}\left|\frac{P(1 / t)}{t^{1-\alpha}}\right|^{q} d t\right)^{1 / q} \\
& =O\left(\frac{1}{P(n)}\right)\left(\int_{1}^{n} \frac{(P(y))^{q}}{y^{(\alpha-1) q}} \cdot \frac{d y}{y^{2}}\right)^{1 / q} \\
& =O\left(\frac{1}{P(n)}\right)\left(\int_{1}^{n} \frac{(P(y))^{q}}{y^{\alpha q-q+2}} d y\right)^{1 / q} \\
& =O\left(\frac{1}{P(n)}\right) \cdot O\left(\frac{P(n)}{n^{\alpha+1 / q-1}}\right) \\
& =O(1 / n-1 / p) .
\end{aligned}
$$

Hence

$(4.4)$

$$
\begin{aligned}
E_{n}^{*}(f) & =\min _{n}\left\|f-N_{n}\right\|_{p} \\
& =o\left(\frac{1}{n^{\alpha-1 / p}}\right) .
\end{aligned}
$$

This completes the proof of Theorem 2 .

5.

As $p \rightarrow \infty$ (and therefore $q=1$ ), Theorem 1 is equivalent to Theorem A, for $0<\alpha<\delta \leq 1$. Also Theorem 2, for $p_{n}=\left(\begin{array}{c}n+\delta-1 \\ \delta-1\end{array}\right)$ is equivalent to Theorem 1 .

\section{References.}

[1] G. Alexits, Convergence problems of orthogonal series (Pergamon Press, New York, Oxford, London, Paris, 1961).

[2] G. Alexits und D. Králik, "Über die Approximation mit starken de la Vallée-Poussinschen Mitteln", Acta Math. Acad. Sci. Hungar. 16 (1965), 43-49. 
[3] G. Alexits und L. Leindler, "Über die Approximation im starken Sinne", Acta Math. Acad. Sci. Hungar. 16 (1965), 27-32.

[4] Masako Izumi and Shin-ichi Izumi, "On the Nörlund summability of Fourier series", Proc. Japan Acad. 45 (1969), 773-778.

[5] G.G. Lorentz, Approximation of functions (Holt, Rinehart \& Winston, New York; Chicago, Illinois; Toronto, Ontario; 1966).

[6] Badri N. Sahney, "On the Nфrlund summability of Fourier series", Canad. J. Math. 22 (1970), 86-91.

[7] A. Zygmund, Trigonometric series, Vol. 1, 2nd ed. (Cambridge University Press, Cambridge, 1959).

Department of Mathematics, Statistics and Computer Science,

The University,

Calgary,

Alberta, Canada;

and

Department of Mathematics,

Institute of Advanced Studies,

Australian National University,

Canberra, ACT. 